\title{
Error Estimates for the Coupling of Analytical and Numerical Solutions
}

\section{Klaus Gürlebeck, Uwe Kähler \& Dmitrii Legatiuk}

\section{Complex Analysis and Operator Theory}

ISSN 1661-8254

Volume 11

Number 5

Complex Anal. Oper. Theory (2017) 11:1221-1240

DOI 10.1007/s11785-016-0583-y

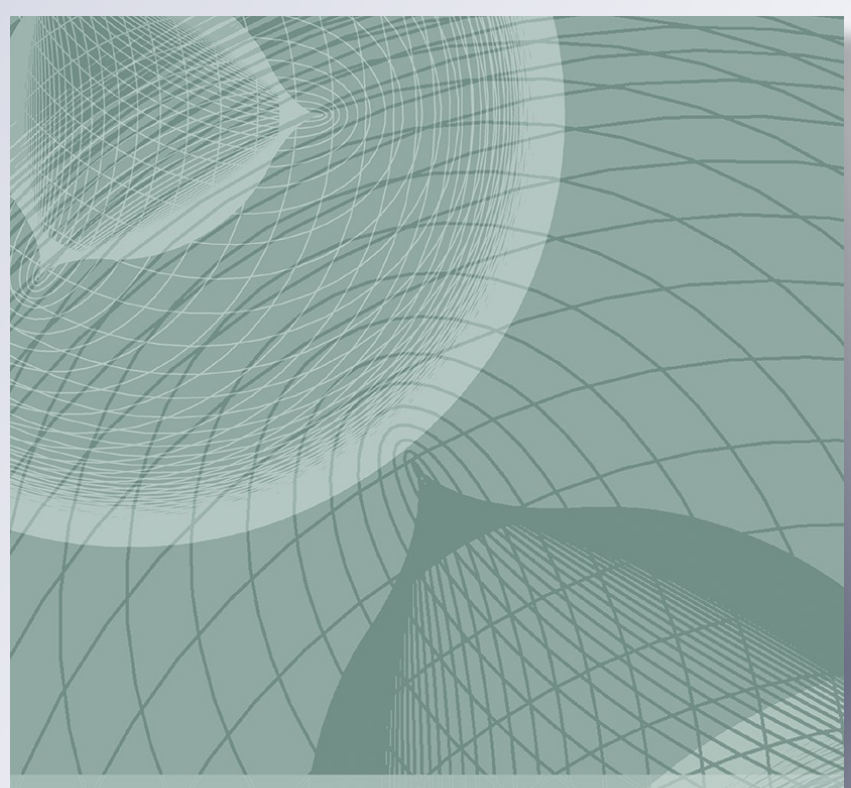

Complex Analysis and Operator Theory

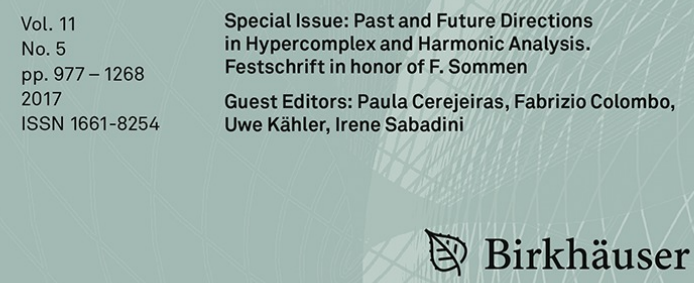


Your article is protected by copyright and all rights are held exclusively by Springer International Publishing. This e-offprint is for personal use only and shall not be selfarchived in electronic repositories. If you wish to self-archive your article, please use the accepted manuscript version for posting on your own website. You may further deposit the accepted manuscript version in any repository, provided it is only made publicly available 12 months after official publication or later and provided acknowledgement is given to the original source of publication and a link is inserted to the published article on Springer's website. The link must be accompanied by the following text: "The final publication is available at link.springer.com". 


\title{
Error Estimates for the Coupling of Analytical and Numerical Solutions
}

\author{
Klaus Gürlebeck ${ }^{1}$ • Uwe Kähler ${ }^{2}$ • Dmitrii Legatiuk ${ }^{3} \mathbb{D}$
}

Received: 9 July 2016 / Accepted: 3 August 2016 / Published online: 10 August 2016

(C) Springer International Publishing 2016

\begin{abstract}
In this paper we present error estimates for a continuous coupling of an analytical and a numerical solution for a boundary value problem with a singularity. A solution of the Lamé-Navier equation with a singularity caused by a crack is considered as an example. The analytical solution near a singularity is constructed by using complex function theory and coupled continuously with the finite element solution. The objective of this paper is to estimate the coupling error, which cannot be covered by the classical theory of the finite element method.
\end{abstract}

Dedicated to Frank Sommen on the occasion of his 60th birthday.

Communicated by Fabrizio Colombo.

The research of U. Kähler is supported by Portuguese funds through the CIDMA, Center for Research and Development in Mathematics and Applications, and the Portuguese Foundation for Science and Technology ("FCT-Fundação para a Ciência e a Tecnologia"), within project UID/MAT/04106/2013. The research of D. Legatiuk is supported by the German Research Foundation (DFG) via Research Training Group "Evaluation of Coupled Numerical Partial Models in Structural Engineering (GRK 1462)", which is gratefully acknowledged.

$凶$ Dmitrii Legatiuk

dmitrii.legatiuk@uni-weimar.de

Klaus Gürlebeck

klaus.guerlebeck@uni-weimar.de

Uwe Kähler

ukaehler@ua.pt

1 Bauhaus-Universität Weimar, 99423 Weimar, Germany

2 CIDMA, Center for R\&D in Mathematics and Applications, Universidade de Aveiro, Aveiro, Portugal

3 Research Training Group 1462, Bauhaus-Universität Weimar, 99425 Weimar, Germany

Birkhäuser 
Keywords Error estimation · Interpolation · Coupling · Analytical solution · Singularity $\cdot$ Kolosov-Muskhelishvili formulae

Mathematics Subject Classification Primary 30G35; Secondary 18 B99

\section{Introduction}

Daily tasks of engineering practice very often lead to problems containing different types of singularities (like for instance cracks, gaps, corners, interfaces between different materials, etc.). To get high quality results for such problems one needs to consider them very carefully due to the singularity. Particularly, if one keeps in mind an application of numerical methods (like finite element method, finite difference method, etc.), then this would lead to the necessity of adapting the numerical scheme. For instance, in mesh-based methods it means one needs to refine the mesh in the region near a singularity.

Another way is to construct an analytical solution near the singularity. Methods of complex function theory are a powerful tool to construct exact solutions for problems of linear elasticity. The Kolosov-Muskhelishvili formulae allow us to describe the near-field solution of a crack tip problem in terms of two holomorphic functions $\Phi(z)$ and $\Psi(z), z \in \mathbb{C}[16]$. The analytical solution based on complex function theory gives us a high accuracy of the solution in the neighbourhood of the singularity. Because of using exact solutions of the partial differential equations all details of the mathematical model are preserved. Particularly, in [14] one can find a remarkable series of results in fracture mechanics, where the important physical quantities are calculated exactly by help of complex function theory.

The disadvantage of the complex analytic approach is that such problem can be solved explicitly only for some elementary (simple) domains. Domains coming from practical engineering problems usually are more complicated. Therefore, a combination of both types of methods is expected to give better results. The problem of such a combination comes from the following facts: on the one hand, the analytical solution which is constructed by complex function theory is given by a series of holomorphic functions, but on the other hand, the standard finite element solution is based on spline functions. Due to this fact the coupling process has to be considered more carefully.

While there are several ways to introduce a coupling between analytical solution and FEM solution possible (see e.g. [10,17-19]), we focus on a continuous coupling in this paper. This coupling was proposed in $[1,13]$. To obtain the continuous coupling a special element is introduced. This element contains an exact solution to the differential equation with the correct singularity and so-called coupling elements. The requirement for these coupling elements is to insure $C^{0}$ continuity for the displacements. In this case we can expect the solution to have a higher regularity than in the case of a simple discrete coupling.

The results obtained in [12] show that an error estimate for the case of a fixed size of the special element can be constructed only by studying the so-called coupling error. The coupling error takes place at the interaction interface between analytical and numerical solutions. This error represents the interpolation error coming from the 
difference between exact boundary data (ideal case, if the finite element solution has no error in the remaining part of the domain) and disturbed boundary data (including error of finite element approximation). In this paper we present an explicit estimate of the coupling error. To make clear where appears the considered interpolation problem we briefly recall the idea of the coupling method.

The proposed two-dimensional coupling is considered as a preliminary study for an extension of such a coupling to three-dimensional problems. This extension is possible due to recently [2-4] introduced spatial analogues of the classical KolosovMuskhelishvili formulae. In this case an analytical solution is constructed in terms of monogenic functions used in representations for components of the displacement field and the stress tensor. But to understand important steps in the coupling process the two-dimensional case has to be studied at first.

\section{The Method of Coupling of an Analytical and a Finite Element Solution}

In this section we briefly recall the main ideas of the coupling method: the general description of a domain and its decomposition, construction of the analytical solution for the crack tip problem, interpolation problem at the interaction interface, and geometrical properties of coupling elements. For more details we refer to $[1,10,13]$.

\subsection{Geometrical Settings and Boundary Value Problem in a Domain}

Let us consider a bounded simply connected domain $\Omega \subset \mathbb{C}$ with a Lipschitz boundary $\Gamma$ containing a crack. To describe the behaviour of the continuum near the crack-tip we are going to model more precisely the near-field domain, called $\Omega_{\mathrm{SE}}$ (see Fig. 1). The domain $\Omega_{\mathrm{SE}}$ is called a special element in the triangulation $\mathcal{F}_{h}$ over the domain $\Omega$. The special element is always located at the crack tip, i.e. at the origin of a Cartesian coordinate system.

In the domain $\Omega_{\mathrm{SE}}$ we distinguish two sub-domains: the discrete "numerical" domain $\Omega_{\mathrm{D}}$ and the "analytical" domain $\Omega_{\mathrm{A}}$. A mesh over $\Omega_{\mathrm{D}}$ is made of two types of elements: the standard triangular elements (elements $A-H$ in Fig. 2) and the coupling

Fig. 1 Domain $\Omega$ with a crack

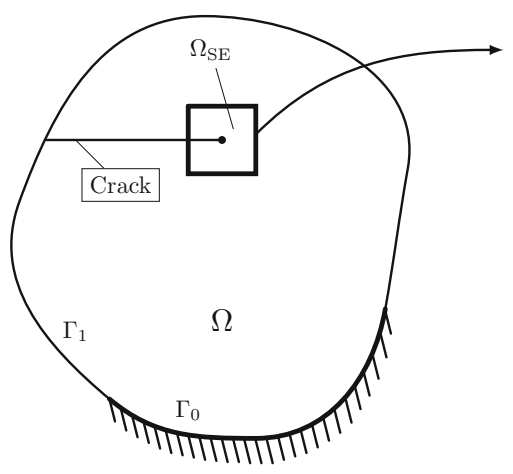


Fig. 2 Special element $\Omega_{\mathrm{SE}}=\boldsymbol{\Omega}_{\mathrm{A}} \cup \boldsymbol{\Omega}_{\mathrm{D}}$

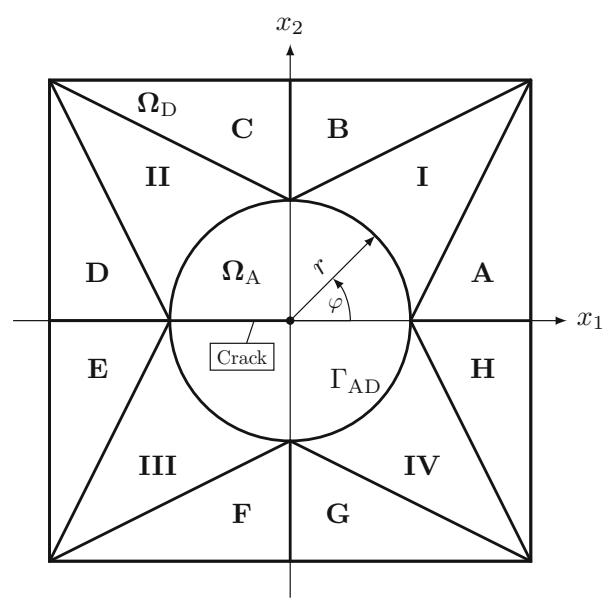

elements (elements $I-I V$ in Fig. 2). The coupling elements serve to couple continuously solutions in $\Omega_{\mathrm{D}}$ and $\Omega_{\mathrm{A}}$. We call the sub-domain $\Omega_{\mathrm{A}}$ analytical in the sense that the constructed solutions are exact solutions to the differential equation in $\Omega_{\mathrm{A}}$. The principle idea behind the special element is to obtain the continuous connection through the whole interaction interface $\Gamma_{\mathrm{AD}}$ by introducing a special interpolation operator (see for example [10,11]).

Finally, we introduce a triangulation $\mathcal{F}_{h}$ over the domain $\Omega$ as follows

$$
\mathcal{F}_{h}=\mathcal{F}_{h}^{\mathbb{A}} \cup \mathcal{F}_{h}^{\mathbb{T}} \cup \mathcal{F}_{h}^{T}
$$

where $\mathcal{F}_{h}^{\mathbb{A}}$ is the set of elements based on the analytical solution in $\Omega_{\mathrm{A}}, \mathcal{F}_{h}^{\mathbb{T}}$ is the set of the coupling elements, and $\mathcal{F}_{h}^{T}$ is the set of the classical elements. These three sets are mutually disjoint and connections between their elements $\mathbb{A}, \mathbb{T}$ and $T$ are defined by common sets of degrees of freedom. Additionally, the connection between $\mathbb{A}$ and $\mathbb{T}$ is supplemented by the continuous coupling through the interface $\Gamma_{\mathrm{AD}}$.

In Fig. 1 the domain $\Omega$ represents a volume which is occupied by a solid body. We consider a classical problem of linear elasticity, which is given by the Lamé-Navier equation together with boundary conditions as follows

$$
\begin{cases}-\mu \Delta \mathbf{u}-(\lambda+\mu) \operatorname{grad} \operatorname{div} \mathbf{u}=\mathbf{f} & \text { in } \Omega, \\ \mathbf{u}=0 & \text { on } \Gamma_{0}, \\ \sum_{j=1}^{3} \sigma_{i j}(\mathbf{u}) n_{j}=g_{i} & \text { on } \Gamma_{1}, \quad 1 \leq i \leq 3,\end{cases}
$$

where $\lambda$ and $\mu$ are material constants, and $\mathbf{f}$ is the density of volume forces, $\vec{u}$ is the unknown displacement vector, $g_{i}$ are components of density of surface forces, $n_{j}$ are components of the unit outer normal vector, and $\sigma_{i j}$ are components of the stress tensor, $\Gamma_{0}$ and $\Gamma_{1}$ are part of the boundary with Dirichlet and Neumann boundary 
conditions, respectively. In this article we concentrate ourselves to the plane strain state, i.e. $u_{3}=0, \varepsilon_{3 j}=0, j=1, \ldots, 3$, for more details we refer to [15].

\subsection{Analytical Solution and Interpolation Problem}

To construct the analytical solution to the crack tip problem we use the KolosovMuskhelishvili formulae in polar coordinates (see [16]), which are given by

$$
\begin{aligned}
2 \mu\left(u_{r}+i u_{\varphi}\right) & =e^{-i \varphi}\left(\kappa \Phi(z)-z \overline{\Phi^{\prime}(z)}-\overline{\Psi(z)}\right), \\
\sigma_{r r}+\sigma_{\varphi \varphi} & =2\left[\Phi^{\prime}(z)+\overline{\Phi^{\prime}(z)}\right], \\
\sigma_{\varphi \varphi}-\sigma_{r r}+2 i \sigma_{r \varphi} & =2 e^{2 i \varphi}\left[\bar{z} \Phi^{\prime \prime}(z)+\Psi^{\prime}(z)\right] .
\end{aligned}
$$

where $\Phi(z)$ and $\Psi(z), z \in \mathbb{C}$, are two holomorphic functions, and the factor $\kappa \in(1,3)$ represents Kolosov's constant.

The functions $\Phi(z)$ and $\Psi(z)$ can be written in terms of power series expansion

$$
\Phi(z)=\sum_{k=0}^{\infty} a_{k} z^{\lambda_{k}}, \quad \Psi(z)=\sum_{k=0}^{\infty} b_{k} z^{\lambda_{k}}
$$

where $a_{k}$ and $b_{k}$ are unknown coefficients which are determined through the boundary conditions for the global problem and the powers $\lambda_{k}$ describe the behaviour of the displacements and stresses near the crack tip and are determined through the boundary conditions on the crack faces.

In the case of traction free boundary conditions on the crack faces the displacement field can be written as follows (for more details see [14])

$$
\begin{aligned}
2 \mu\left(u_{1}+i u_{2}\right)= & \sum_{n=0,2, \ldots}^{\infty} r^{\frac{n}{2}}\left[a_{n}\left(\kappa e^{i \varphi \frac{n}{2}}+e^{-i \varphi \frac{n}{2}}\right)\right. \\
& \left.+\frac{n}{2} \bar{a}_{n}\left(e^{-i \varphi \frac{n}{2}}-e^{-i \varphi\left(\frac{n}{2}-2\right)}\right)\right] \\
& +\sum_{n=1,3, \ldots}^{\infty} r^{\frac{n}{2}}\left[a_{n}\left(\kappa e^{i \varphi \frac{n}{2}}-e^{-i \varphi \frac{n}{2}}\right)\right. \\
& \left.+\frac{n}{2} \bar{a}_{n}\left(e^{-i \varphi \frac{n}{2}}-e^{-i \varphi\left(\frac{n}{2}-2\right)}\right)\right] .
\end{aligned}
$$

The displacement field (2.2) satisfies all the conditions on the crack faces. The asymptotic behaviour at the crack tip is controlled by half-integer powers [14].

To get a continuous displacement field through the boundary $\Gamma_{\mathrm{AD}}$ we introduce a special interpolation operator. The unique solvability of the corresponding interpolation problem is proved in $[10,11]$. How to get a continuous coupling is presented in [13], and we will not repeat it here. Let us consider $n$ nodes on the interface $\Gamma_{\mathrm{AD}}$ belonging to the interval $[-\pi, \pi]$ (see Fig. 3). 
Fig. 3 Nodes and coupling elements

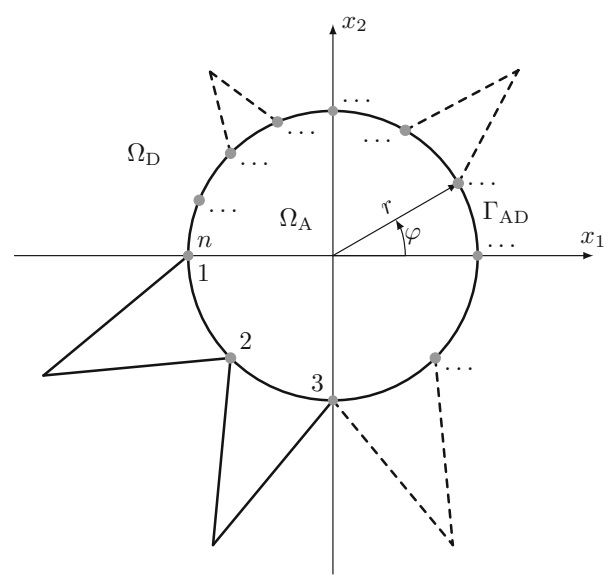

To interpolate at the interface $\Gamma_{\mathrm{AD}}$ we truncate the analytical solution (2.2). Therefore we obtain the interpolation function $f_{n}(\varphi)$ restricted to the interface $\Gamma_{\mathrm{AD}}\left(r=r_{\mathrm{A}}\right)$ in the following form

$$
\begin{aligned}
f_{n}(\varphi)= & \sum_{k=0,2, \ldots}^{N_{1}} r_{\mathrm{A}}^{\frac{k}{2}}\left[a_{k}\left(\kappa e^{i \varphi \frac{k}{2}}+e^{-i \varphi \frac{k}{2}}\right)+\frac{k}{2} \bar{a}_{k}\left(e^{-i \varphi \frac{k}{2}}-e^{-i \varphi\left(\frac{k}{2}-2\right)}\right)\right] \\
& +\sum_{k=1,3, \ldots}^{N_{2}} r_{\mathrm{A}}^{\frac{k}{2}}\left[a_{k}\left(\kappa e^{i \varphi \frac{k}{2}}-e^{-i \varphi \frac{k}{2}}\right)+\frac{k}{2} \bar{a}_{k}\left(e^{-i \varphi \frac{k}{2}}-e^{-i \varphi\left(\frac{k}{2}-2\right)}\right)\right],
\end{aligned}
$$

where the numbers of basis functions $N_{1}$ and $N_{2}$ are related to $n$ as follows:

$$
\begin{aligned}
& N_{1}=n-m, \quad \text { with } \begin{cases}m=2 & \text { for even } n, \\
m=1 & \text { for odd } n,\end{cases} \\
& N_{2}=n-m, \quad \text { with } \begin{cases}m=1 & \text { for even } n, \\
m=2 & \text { for odd } n .\end{cases}
\end{aligned}
$$

To obtain the basis functions for finite element approximation we interpolate the unknown displacements $\mathbf{U}_{j}, j=0, \ldots, n-1$ at the interface $\Gamma_{\mathrm{AD}}$.

\subsection{Geometry of the Coupling Elements}

In this section we briefly introduce the basic properties of the coupling elements. Geometrically we define a single coupling element $\mathbb{T}$ as a triangle with three vertices $v_{1}, v_{2}, v_{3}$ which has a curved edge $\left\langle v_{3}, v_{1}\right\rangle$. As it can be seen in Fig. 4 the vertex $v_{2}$ is located at the circle of a radius $R$, which is defined by

$$
R=\frac{r_{\mathrm{A}}+l}{\cos \alpha}
$$


Fig. 4 Geometry of a coupling element

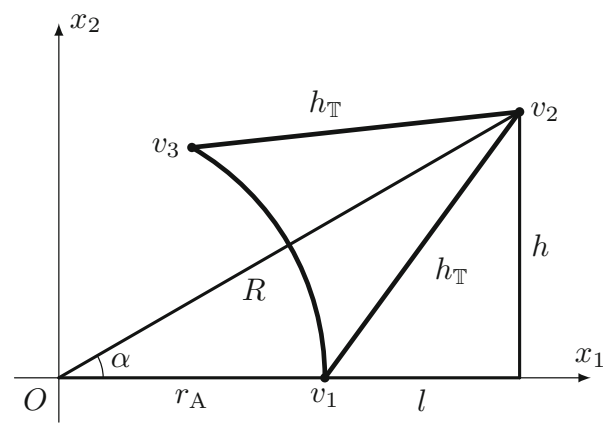

where $\alpha$ is an angle between the line $O v_{2}$, which is the bisector of $\angle\left(v_{1} v_{2} v_{3}\right)$, and $x_{1}$ axis, and $n$ is the number of interpolation nodes. To have a general description of a geometry of a coupling element we perform a rotation of each coupling element in a way to get the orientation shown in Fig. 4.

The angle $\alpha$ in general can vary from element to element depending on a given distribution of nodes. For example, for the equidistant nodes it is given by

$$
\alpha=\frac{\pi}{n-1}
$$

In a general case for a given triangulation $\mathcal{F}_{h}$ geometry of all coupling elements is defined by a set of all angles $\alpha=\left\{\alpha_{1}, \alpha_{2}, \ldots, \alpha_{n-1}\right\}$. In this case we can define an element mesh size of the coupling element $h_{\mathbb{T}}^{(i)}=\operatorname{diam}\left(\mathbb{T}_{i}\right)$ as follows

$$
h_{\mathbb{T}}^{(i)}=\sqrt{\left(r_{\mathrm{A}}+l\right)^{2} \tan ^{2}\left(\alpha_{i}\right)+l^{2}} .
$$

The parameter $h$ in Fig. 4 represents a characteristic size of standard elements neighbouring the coupling element. This characteristic size can be expressed in terms of angles $\alpha_{i}$ as follows

$$
h_{i}=\tan \left(\alpha_{i}\right)\left(r_{\mathrm{A}}+l\right), \quad i=1, \ldots, n-1
$$

For purpose of a global error estimation we assume that the triangulation $\mathcal{F}_{h}$ over the domain $\Omega$ is constructed in a way that the characteristic size of standard elements in the far field of the special element can be expressed by

$$
h=C \max \left(h_{i}\right), \quad i=1, \ldots, n-1,
$$

where $C$ is an arbitrary constant.

The increasing number of nodes at the interface $\Gamma_{\mathrm{AD}}$ leads to a greater number of coupling elements, see Fig. 3. To avoid numerical problems caused by too narrow coupling elements we use the shape parameter criteria according to [20]. The idea is that with the increasing number $n$ we decrease the length of $l$, like it is shown in Fig. 3. 
In that case we can say that the characteristic sizes $h_{\mathbb{T}}$ and $h$ tend to zero with increasing the number of the interpolation nodes at the interface $\Gamma_{\mathrm{AD}}$. Expressions (2.4) and (2.5) for mesh sizes can be used for both strategies of error estimation, which are introduced in the next section.

\section{Estimation of Interpolation Error}

In this section we construct the error estimate for an interpolation problem with basis functions in the form (2.3). Due to the flexibility provided by the construction of the coupling two different strategies for a numerical realisation are possible:

(i) classical realisation of the FEM with a global refinement;

(ii) numerical calculations with a fixed radius of the analytical domain $\boldsymbol{\Omega}_{\mathrm{A}}$.

These two strategies require different approaches to convergence analysis and error estimation of the proposed method.

First strategy leads to a convergence analysis in the framework of the classical theory of the finite element method developed in [5]. But for the proposed method this theory cannot be applied directly due to the following problems: the regularity of basis functions is restricted by the singular term in the analytical solution (2.2) and the coupling elements are not affine-equivalent to each other. We refer to [12] for a complete discussion on how to overcome these problem.

The objective of the this paper is to estimate the coupling error appearing in the second strategy. The idea of the second strategy of a fixed radius $r_{\mathrm{A}}$ is motivated by a practical reason, because if $r_{\mathrm{A}}$ tends to zero we loose the advantage of the correct approximation of the singular solution. Contrary to it from fixed radius and greater number of the coupling elements one can expect "better" approximation of the boundary values for the analytical solution which could lead to higher quality of results near the singularity.

In this case the error over the coupling elements can be obtained by direct application of the results from [5] since the coupling elements are free of singular functions. For the details we refer again to [12]. But construction of the error estimate in the analytical element $\mathbb{A}$ is more complicated, since the radius of $\boldsymbol{\Omega}_{\mathrm{A}}$ is fixed and cannot approach zero. To get the desired estimate we consider the task of approximation by interpolation in our settings:

What is the interpolation error?

To study this question let us consider the following interpolation problem at the interface $\Gamma_{\mathrm{AD}}$

$$
f_{n}\left(\varphi_{j}\right)=\mathbf{U}_{j}, \quad j=0, \ldots, n-1,
$$

where $\varphi_{j} \in[-\pi, \pi]$ are interpolation nodes, which can be arbitrary but mutually different, $\mathbf{U}_{j}$ are displacements at the interpolation nodes.

Interpolation problem (3.1) represents the ideal case when the right hand side is calculated exactly, i.e. $\mathbf{U}_{j}=\mathbf{u}\left(r_{\mathrm{A}}, \varphi_{j}\right)$, where $\mathbf{u}\left(r_{A}, \varphi\right)$ is the exact solution in $\Omega_{\mathrm{A}}$ restricted to the interface $\Gamma_{\mathrm{AD}}$. Since the displacements $\mathbf{U}_{j}$ are determined through the solution of a global boundary value problem in $\Omega$, it means that they are approximated 
by the finite element solution. Thus in reality we have the following interpolation problem

$$
\tilde{f}_{n}\left(\varphi_{j}\right)=\tilde{\mathbf{U}}_{j}, \quad j=0, \ldots, n-1,
$$

where $\tilde{\mathbf{U}}_{j}$ denotes the approximated displacement values.

In the sequel we will refer to interpolation problem (3.1) as to the "exact" interpolation problem, and to interpolation problem (3.2) as to the "disturbed" interpolation problem. We will denote by $f_{n}(\varphi)$ and $\tilde{f}_{n}(\varphi)$ the "exact" and the "disturbed" interpolation solution, correspondingly. Our goal here is to estimate the error between exact and "disturbed" interpolation solutions, i.e.

$$
\left|\mathbf{u}\left(r_{A}, \varphi\right)-\tilde{f}_{n}(\varphi)\right|
$$

Let us rewrite this error as follows

$$
\left|\mathbf{u}\left(r_{A}, \varphi\right)-\tilde{f}_{n}(\varphi)\right| \leq\left|\mathbf{u}\left(r_{A}, \varphi\right)-f_{n}(\varphi)\right|+\left|f_{n}(\varphi)-\tilde{f}_{n}(\varphi)\right|,
$$

where $\left|\mathbf{u}\left(r_{A}, \varphi\right)-f_{n}(\varphi)\right|$ is the interpolation error, and $\left|f_{n}(\varphi)-\tilde{f}_{n}(\varphi)\right|$ is the coupling error. The coupling error represents the difference between interpolation with exact and disturbed values which we have in reality. Following ideas from [6,9] the interpolation error has been constructed in [12] in the following form

$$
\left|\mathbf{u}\left(r_{A}, \varphi\right)-f_{n}(\varphi)\right| \leq \frac{M\left(r_{A}+j h\right)}{j h-2 \varepsilon}\left(\frac{2 r_{\mathrm{A}}}{j h}\right)^{n+1}
$$

where $j>1$ is a scaling factor, $\varepsilon>0$ is a parameter for estimate, for more details we refer to [12].

The main concern of the next section is a construction of the coupling error $\mid f_{n}(\varphi)-$ $\tilde{f}_{n}(\varphi) \mid$.

\subsection{Coupling Error}

Our interest now is to estimate the difference $\left|f_{n}(\varphi)-\tilde{f}_{n}(\varphi)\right|$ between the "approximated" and the "exact" interpolation function. Let us denote by $\boldsymbol{\Phi}=\left\{\Phi_{1}, \Phi_{2}, \ldots, \Phi_{n}\right\}$ a vector of the basis functions corresponding to (2.3), then the "approximated" and the "exact" interpolation functions are given by

$$
f_{n}(\varphi)=[\mathbf{a}]^{T} \boldsymbol{\Phi}, \quad \tilde{f}_{n}(\varphi)=[\tilde{\mathbf{a}}]^{T} \boldsymbol{\Phi},
$$

where $[\mathbf{a}]$ and $[\tilde{\mathbf{a}}]$ are the vectors of unknown coefficients corresponding to the interpolation problems (3.1)-(3.2), respectively. These vectors of the unknown coefficients can be expressed as follows

$$
\mathbf{a}=\left[\Phi_{k}\left(\varphi_{j}\right)\right]^{-1} \mathbf{U}, \quad \tilde{\mathbf{a}}=\left[\Phi_{k}\left(\varphi_{j}\right)\right]^{-1} \tilde{\mathbf{U}}
$$


where $\mathbf{U}$ and $\tilde{\mathbf{U}}$ are vectors of the "exact" and the "disturbed" displacements. Let us introduce the following notation

$$
\mathbf{G}=[\mathrm{G}]_{k j}=\left[\Phi_{k}\left(\varphi_{j}\right)\right]^{-1}
$$

for the inverse of the interpolation matrix. Now we can write

$$
\begin{aligned}
\left|f_{n}(\varphi)-\tilde{f}_{n}(\varphi)\right| & =\left|[\mathbf{a}]^{T} \boldsymbol{\Phi}-[\tilde{\mathbf{a}}]^{T} \boldsymbol{\Phi}\right|=\left|[\mathbf{G} \mathbf{U}]^{T} \boldsymbol{\Phi}-[\mathbf{G} \tilde{\mathbf{U}}]^{T} \boldsymbol{\Phi}\right| \\
& =\left|\mathbf{U}^{T} \mathbf{G}^{T} \boldsymbol{\Phi}-\tilde{\mathbf{U}}^{T} \mathbf{G}^{T} \boldsymbol{\Phi}\right|=\left|\left(\mathbf{U}^{T}-\tilde{\mathbf{U}}^{T}\right) \mathbf{G}^{T} \boldsymbol{\Phi}\right|
\end{aligned}
$$

Let us introduce the constant $\delta^{*}$ as follows

$$
\left|\mathbf{U}_{j}-\tilde{\mathbf{U}}_{j}\right| \leq \delta^{*}, \quad j=0, \ldots, n-1 .
$$

This inequality implies that the coupling error explicitly depends only on the quality of approximation for the displacements at the interface $\Gamma_{\mathrm{AD}}$, which is seems to be natural. We have

$$
\left|f_{n}(\varphi)-\tilde{f}_{n}(\varphi)\right| \leq \delta^{*}\left|\mathbf{G}^{T} \boldsymbol{\Phi}\right|,
$$

where we identify vectors with complex numbers. Thus we need to estimate the transposed inverse of interpolation matrix $\mathbf{G}^{T}$, the vector of the basis functions $\boldsymbol{\Phi}$, and the error $\delta^{*}$.

We start the construction of the coupling estimate by considering the matrix $[\mathrm{G}]_{k j}$. As an estimate we will use the spectral or Frobenius norm of the matrix. The matrix $\left[\Phi_{k}\left(\varphi_{j}\right)\right]^{-1}$ obtained from the basis function in the form (2.3) is not diagonally dominant and, therefore, its Gerschgorin disks will not give us the isolated regions for the eigenvalues. To overcome this problem we will work with the modified interpolation function in the form

$$
f_{n}(\varphi)=\sum_{k=0}^{n-1} c_{k} e^{i \varphi \frac{k}{2}}
$$

In [11] it was shown that the modified interpolation function can be obtained from the original function (2.3) via an almost diagonal transformation matrix $M$. Components of the transformation matrix $M$ in the real form are given explicitly by

$$
\begin{aligned}
M_{1,1} & =M_{2,2}=\kappa+1, & M_{7,3} & =-\frac{1}{2} r_{\mathrm{A}}^{\frac{1}{2}}, \\
M_{3,3} & =M_{4,4}=\kappa r_{\mathrm{A}}^{\frac{1}{2}}, & M_{8,4} & =\frac{1}{2} r_{\mathrm{A}}^{\frac{1}{2}}, \\
M_{5,5} & =r_{\mathrm{A}}(\kappa-1), & M_{3,7} & =-\frac{3}{2} r_{\mathrm{A}}^{\frac{1}{2}},
\end{aligned}
$$




$$
\begin{gathered}
M_{6,6}=r_{\mathrm{A}}(\kappa+1), \quad M_{4,8}=\frac{3}{2} r_{\mathrm{A}}^{\frac{1}{2}}, \\
M_{j, j}=M_{j+1, j+1}=\kappa r_{\mathrm{A}}^{\frac{j-4}{2}}, \quad j=7,9, \ldots, n, \\
M_{j+1, j+1}=M_{j+2, j+2}=\kappa r_{\mathrm{A}}^{\frac{j-5}{2}}, j=8,10, \ldots, n,
\end{gathered}
$$

while other entries are zero. The transformation matrix allows us to calculate uniquely the coefficients $a_{k}$ and $\bar{a}_{k}$ from the new coefficients $c_{k}$. Therefore, as it was shown in [11], we can work with the equivalent interpolation problem

$$
\sum_{k=0}^{n-1} c_{k} e^{i \varphi_{j} \frac{k}{2}}=\mathbf{U}_{j}, \quad j=0, \ldots, n-1 .
$$

The matrix of this interpolation problem is a Vandermonde matrix, which we denote by $F$. This allows us to work instead of the matrix $\left[\Phi_{k}\left(\varphi_{j}\right)\right]^{-1}$ with the transformation matrix $M$ and the Vandermonde matrix $F$. In this case the coefficients $a_{k}$ and $\tilde{a}_{k}$ can be calculated as follows

$$
a=M^{-1} F^{-1} \mathbf{U}, \quad \tilde{a}=M^{-1} F^{-1} \tilde{\mathbf{U}}
$$

and estimate (3.4) has the following form

$$
\left|f_{n}(\varphi)-\tilde{f}_{n}(\varphi)\right| \leq \delta^{*}\left|M^{-1} F^{-1} \boldsymbol{\Phi}\right| \leq \delta^{*}\left\|M^{-1}\right\|_{2}\left\|F^{-1}\right\|_{2}\|\boldsymbol{\Phi}\|_{2}
$$

and the remaining task is to estimate the spectral norms of $M^{-1}$ and $F^{-1}$ as well as the norm of $\boldsymbol{\Phi}$.

\subsubsection{Spectral Norm of the Transformation Matrix}

Let us at first study the matrix $M$. The spectral norm of the inverse matrix can be calculated as follows

$$
\left\|M^{-1}\right\|_{2}=\sqrt{\left(\min _{1 \leq j \leq n}\left|\lambda_{j}\right|\right)^{-1}}
$$

where $\lambda_{1}, \lambda_{2}, \ldots, \lambda_{n}$ are the eigenvalues of $M M^{T}$.

Taking into account the structure of the matrix $M$ (3.5) one can verify by straightforward calculations, that the matrix $M M^{T}$ has an almost diagonal structure. Elements of the matrix $M M^{T}$ are explicitly given as follows

$$
\begin{aligned}
& {\left[M M^{T}\right]_{j, j}=\left(M_{j, j}\right)^{2} \text { for } j=1,2,11,12, \ldots, 2 n,} \\
& {\left[M M^{T}\right]_{3,3}=\left[M M^{T}\right]_{4,4}=\frac{1}{4} r_{\mathrm{A}}\left(4 \kappa^{2}+9 r_{\mathrm{A}}^{2}\right),}
\end{aligned}
$$




$$
\begin{aligned}
{\left[M M^{T}\right]_{5,5} } & =r_{\mathrm{A}}^{2}\left(\kappa^{2}-2 \kappa+1+4 r_{\mathrm{A}}^{2}\right), \\
{\left[M M^{T}\right]_{6,6} } & =r_{\mathrm{A}}^{2}\left(\kappa^{2}+2 \kappa+1+4 r_{\mathrm{A}}^{2}\right), \\
{\left[M M^{T}\right]_{7,7} } & =\left[M M^{T}\right]_{8,8}=\frac{1}{4} r_{\mathrm{A}}\left(1+4 \kappa^{2} r_{\mathrm{A}}^{2}\right), \\
{\left[M M^{T}\right]_{9,9} } & =\left[M M^{T}\right]_{10,10}=r_{\mathrm{A}}^{4} \kappa^{2}, \\
{\left[M M^{T}\right]_{7,3} } & =\left[M M^{T}\right]_{3,7}=-\frac{1}{2} \kappa r_{\mathrm{A}}\left(1+3 r_{\mathrm{A}}^{2}\right), \\
{\left[M M^{T}\right]_{8,4} } & =\left[M M^{T}\right]_{4,8}=\frac{1}{2} \kappa r_{\mathrm{A}}\left(1+3 r_{\mathrm{A}}^{2}\right), \\
{\left[M M^{T}\right]_{9,5} } & =\left[M M^{T}\right]_{5,9}=-2 \kappa r_{\mathrm{A}}^{4}, \\
{\left[M M^{T}\right]_{10,6} } & =\left[M M^{T}\right]_{6,10}=2 \kappa r_{\mathrm{A}}^{4} .
\end{aligned}
$$

Thus the $2 n-8$ eigenvalues of matrix (3.7) are given by

$$
\lambda_{j}=\left(M_{j j}\right)^{2}, \quad j=1,2,11,12, \ldots, 2 n,
$$

or by taking into account the form of elements $M_{j j}$

$$
\lambda_{1,2}=(\kappa+1)^{2}, \quad \lambda_{j}=\left\{\begin{array}{ll}
\kappa^{2} r_{\mathrm{A}}^{\frac{j-1}{2}}, & \text { if } j \text { is odd, } \\
\kappa^{2} r_{\mathrm{A}}^{\frac{j-2}{2}}, & \text { if } j \text { is even, }
\end{array} \quad j=11, \ldots, 2 n .\right.
$$

The remaining eight eigenvalues are the eigenvalues of the non-diagonal sub-matrix of matrix (3.7) for $j, k=3, \ldots, 10$. As we can see the eigenvalues $\lambda_{j}, j=1, \ldots, 2 n$ depend on the only two parameters $r_{\mathrm{A}}$ and $\kappa$. Since the parameter $r_{\mathrm{A}}$ is the radius of the analytical domain $\Omega_{\mathrm{A}}$ for practical calculations we can always use a normalised radius, i.e. $r_{\mathrm{A}}=1$. For the reader interested in dependence of the eigenvalues on $r_{\mathrm{A}}$ we refer to [13]. For the normalised radius all possible eigenvalues have the following form

$$
\begin{aligned}
\lambda_{1,2} & =(\kappa+1)^{2}, \\
\lambda_{3} & =\kappa^{2}+\kappa+\frac{5}{2}+\frac{1}{2} \sqrt{20 \kappa^{2}+20 \kappa+25}, \\
\lambda_{4} & =\kappa^{2}+\kappa+\frac{5}{2}-\frac{1}{2} \sqrt{20 \kappa^{2}+20 \kappa+25}, \\
\lambda_{5} & =\kappa^{2}-\kappa+\frac{5}{2}+\frac{1}{2} \sqrt{20 \kappa^{2}-20 \kappa+25}, \\
\lambda_{6} & =\kappa^{2}-\kappa+\frac{5}{2}-\frac{1}{2} \sqrt{20 \kappa^{2}-20 \kappa+25}, \\
\lambda_{7,9} & =\frac{5}{4}+\kappa^{2}+\frac{1}{8} \sqrt{256 \kappa^{2}+64},
\end{aligned}
$$




$$
\begin{aligned}
\lambda_{8,10} & =\frac{5}{4}+\kappa^{2}-\frac{1}{8} \sqrt{256 \kappa^{2}+64}, \\
\lambda_{j} & =\kappa^{2}, \quad j=11, \ldots, 2 n .
\end{aligned}
$$

Taking into account that $\kappa$ is a material constant depending on Poisson's ratio, it's easy to check that these eigenvalues are ordered as follows

$$
\lambda_{6}<\lambda_{8,10}<\lambda_{4}<\lambda_{j}<\lambda_{1,2,5,7,9}<\lambda_{3}
$$

Therefore the smallest eigenvalue for $\kappa \in(1,3)$ is $\lambda_{6}$. Thus the spectral norm of $M^{-1}$ is given by

$$
\left\|M^{-1}\right\|_{2}=\sqrt{\lambda_{6}^{-1}}=\frac{1}{\sqrt{\kappa^{2}-\kappa+\frac{5}{2}-\frac{1}{2} \sqrt{20 \kappa^{2}-20 \kappa+25}}} .
$$

The obtained estimate for the spectral norm of $M^{-1}$ is uniform, i.e. it is independent on the number of interpolation nodes $n$.

\subsubsection{Spectral Norm of the Vandermonde Matrix}

Now we will construct the estimate for the spectral norm of $F^{-1}$, where the matrix $F$ is a Vandermonde matrix. If we consider the interpolation problem (3.6) for arbitrary interpolation nodes $\varphi_{j} \in[-\pi, \pi]$ then the entries of the matrix $F$ can be written as

$$
F_{\varphi_{j} k}=e^{\frac{1}{2} i \frac{2 \pi}{n-1} \varphi_{j} k}, \quad j=-\frac{n-1}{2}, \ldots, \frac{n-1}{2}, \quad k=0, \ldots, n-1 .
$$

To construct the estimate for the spectral norm of $F^{-1}$ we will work with the conjugate of the interpolation matrix and, additionally, we rescale the entries of the matrix as follows

$$
T_{j k}:=\frac{1}{\sqrt{n}} e^{-\frac{1}{2} i \frac{2 \pi}{n-1} \varphi_{j} k}
$$

This matrix is also a Vandermonde matrix, and it can be considered as a Fourier matrix for a signal $x$. Let $\hat{x}$ be its Fourier transform, defined by $\hat{x}=T x$. We will use this relation to the Finite Fourier Transform later on in the construction of the estimate.

Our goal here is to get estimates for the eigenvalues of the matrix $T T^{*}$, so we have

$$
\left(T T^{*}\right)_{j k}=\sum_{m=0}^{n-1} T_{\varphi_{j} m} T_{m \varphi_{k}}^{*}=\frac{1}{n} \sum_{m=0}^{n-1} \omega^{\frac{1}{2}\left(\varphi_{j}-\varphi_{k}\right) m}
$$

where

$$
\omega=e^{-i \frac{2 \pi}{n-1}}
$$


Following [8] we rewrite the matrix $T T^{*}$ as follows

$$
N=\left[N_{p q}\right]_{p, q=0}^{n-1}=\left[s\left(\varphi_{p}-\varphi_{q}\right)\right]_{p, q=0}^{n-1},
$$

where

$$
s(x)=\frac{1}{n} \sum_{k=0}^{n-1} \omega^{\frac{1}{2} k x} .
$$

The introduced matrix $N$ is not diagonally dominant, therefore, its Gershgorin disks are not suitable for estimating for the eigenvalues. To overcome this problem we introduce additionally to the matrix $N$ and function $s(x)$ two functions $s^{+}(x)$ and $s^{-}(x)$, such that their Finite Fourier Transforms $\hat{s}^{+}(x), \hat{s}^{-}(x)$ satisfy the following conditions

$$
\hat{s}^{-}(x) \in \mathbb{R}, \quad \hat{s}^{+}(x) \in \mathbb{R}, \quad 0 \leq \hat{s}^{-}(x) \leq \hat{s}(x) \leq \hat{s}^{+}(x) .
$$

The matrices corresponding to $\hat{s}^{-}(x)$ and $\hat{s}^{+}(x)$ are defined by

$$
\begin{aligned}
& N^{+}=\left[N_{p q}^{+}\right]_{p, q=0}^{n-1}=\left[s^{+}\left(\varphi_{p}-\varphi_{q}\right)\right]_{p, q=0}^{n-1}, \\
& N^{-}=\left[N_{p q}^{-}\right]_{p, q=0}^{n-1}=\left[s^{-}\left(\varphi_{p}-\varphi_{q}\right)\right]_{p, q=0}^{n-1} .
\end{aligned}
$$

The matrices $N^{-}, N$, and $N^{+}$are positive semi-definite and they can be ordered as follows

$$
0 \leq N^{-} \leq N \leq N^{+}
$$

This means that we can get estimates for the greatest and the smallest eigenvalues of the matrix $N$ by

$$
\begin{aligned}
\lambda_{\max }\left(N^{-}\right) & \leq \lambda_{\max }(N) \leq \lambda_{\max }\left(N^{+}\right), \\
\lambda_{\min }\left(N^{-}\right) & \leq \lambda_{\min }(N) \leq \lambda_{\min }\left(N^{+}\right) .
\end{aligned}
$$

The Gerschgorin discs of the matrix $N$ are given by

$$
D_{j}=\left\{z \in \mathbb{C}:\left|z-N_{j j}\right| \leq R_{j}(N)\right\},
$$

where

$$
N_{j j}=s(0)=n, \quad R_{j}(N)=\sum_{\substack{k=0, k \neq j}}^{n-1}\left|N_{j k}\right| .
$$


As we have mentioned above the matrix $N$ is not diagonally dominant, therefore instead of working with its Gershgorin disk we will work with the Gerschgorin disks of $N^{-}$and $N^{+}$. Using the bounds (3.9) we can estimate

$$
\begin{aligned}
& \lambda_{\max }(N) \leq \lambda_{\max }\left(N^{+}\right) \leq s^{+}(0)+\max _{j} R_{j}\left(N^{+}\right), \\
& \lambda_{\min }(N) \geq \lambda_{\min }\left(N^{-}\right) \geq s^{-}(0)-\max _{j} R_{j}\left(N^{-}\right) .
\end{aligned}
$$

An important task now is to choose two functions $s^{+}(x)$ and $s^{-}(x)$ such that the conditions (3.8) are satisfied. Following [8] we introduce a family of signals $V(k ; a, b, t)$, periodic in $k$ with period $n$, whose Finite Fourier Transforms $\hat{V}(k ; a, b, t)$ are real and trapezoidal

$$
\hat{V}(k ; a, b, t)= \begin{cases}\frac{k-a+t}{t}, & k \in[a-t+1, a-t], \\ 1, & k \in[a, b], \\ \frac{t-k+b}{t}, & k \in[b+1, b+t-1] .\end{cases}
$$

The inverse Finite Fourier Transform of the trapezoidal function (3.10) is given by the expression

$$
\begin{aligned}
V(k ; a, b, t) & =\frac{1}{\sqrt{n}} \sum_{j=-\frac{n-1}{2}}^{\frac{n-1}{2}} \hat{V}(j ; a, b, t) e^{\frac{1}{2} i \frac{2 \pi}{n-1} j k} \\
& =e^{i \frac{\pi k}{2(n-1)}(a+b)} \frac{\sin \left(\frac{\pi k t}{2(n-1)}\right) \sin \left(\frac{\pi k}{2(n-1)}(b-a+t)\right)}{t \sqrt{n} \sin ^{2}\left(\frac{\pi k}{2(n-1)}\right)} .
\end{aligned}
$$

As functions $s^{+}(k)$ and $s^{-}(k)$ we choose the following functions

$$
\begin{aligned}
& s^{+}(k)=\frac{1}{\sqrt{n}} V\left(k ;-\frac{n-1}{2}, \frac{n-1}{2}, t\right)=\frac{\sin \left(\frac{\pi k t}{2(n-1)}\right) \sin \left(\frac{\pi k}{2(n-1)}(n-1+t)\right)}{t n \sin ^{2}\left(\frac{\pi k}{2(n-1)}\right)}, \\
& s^{-}(k)=\frac{1}{\sqrt{n}} V\left(k ;-\frac{n-1}{2}+t, \frac{n-1}{2}-t, t\right)=\frac{\sin \left(\frac{\pi k t}{2(n-1)}\right) \sin \left(\frac{\pi k}{2(n-1)}(n-1-t)\right)}{t n \sin ^{2}\left(\frac{\pi k}{2(n-1)}\right)},
\end{aligned}
$$

and

$$
s^{+}(0)=\frac{n-1+t}{n}, \quad s^{-}(0)=\frac{n-1-t}{n} .
$$


Let us denote by $D$ the maximum distance between two nodes, and by $d$ the minimum distance, i.e.

$$
d \leq\left|\varphi_{p}-\varphi_{q}\right| \leq D, \quad p \neq q .
$$

To obtain the estimates for the eigenvalues of the original matrix, at first we need to get the Gerschgorin disks for the matrix associated with the function $V(k ; a, b, t)$. For this matrix we have the following estimate

$$
\begin{aligned}
& \sum_{\substack{p=0, p \neq q}}^{n-1} \frac{1}{\sqrt{n}}\left|V\left(\varphi_{p}-\varphi_{q} ; a, b, t\right)\right| \\
& \quad=\sum_{\substack{p=0, p \neq q}}^{n-1}\left|e^{i \frac{\pi k}{2(n-1)}(a+b)} \frac{\sin \left(\frac{\pi\left(\varphi_{p}-\varphi_{q}\right) t}{2(n-1)}\right) \sin \left(\frac{\pi\left(\varphi_{p}-\varphi_{q}\right)}{2(n-1)}(b-a+t)\right)}{n t \sin ^{2}\left(\frac{\pi\left(\varphi_{p}-\varphi_{q}\right)}{2(n-1)}\right)}\right| \\
& \leq \sum_{\substack{p=0, p \neq q}}^{n-1}\left|\frac{\sin \left(\frac{\pi\left(\varphi_{p}-\varphi_{q}\right) t}{2(n-1)}\right) \sin \left(\frac{\pi\left(\varphi_{p}-\varphi_{q}\right)}{2(n-1)}(b-a+t)\right)}{n t \sin ^{2}\left(\frac{\pi\left(\varphi_{p}-\varphi_{q}\right)}{2(n-1)}\right)}\right| \\
& =\sum_{\substack{p=0, p \neq q}}^{n-1}\left|\frac{\frac{1}{2}\left(\cos \left[\frac{\pi\left(\varphi_{p}-\varphi_{q}\right)}{2(n-1)}(b-a)\right]-\cos \left[\frac{\pi\left(\varphi_{p}-\varphi_{q}\right)}{2(n-1)}(b-a+2 t)\right]\right)}{n t \sin ^{2}\left(\frac{\pi\left(\varphi_{p}-\varphi_{q}\right)}{2(n-1)}\right)}\right| .
\end{aligned}
$$

Expanding the numerator in the last fraction into its Taylor series and taking into account that the higher order terms are decreasing with order $n^{2 k+1}$ for an increasing value of $n$, we obtain the following estimate

$$
\begin{gathered}
\sum_{\substack{p=0, p \neq q}}^{n-1}\left|\frac{\frac{1}{2}\left(\cos \left[\frac{\pi\left(\varphi_{p}-\varphi_{q}\right)}{2(n-1)}(b-a)\right]-\cos \left[\frac{\pi\left(\varphi_{p}-\varphi_{q}\right)}{2(n-1)}(b-a+2 t)\right]\right)}{n t \sin ^{2}\left(\frac{\pi\left(\varphi_{p}-\varphi_{q}\right)}{2(n-1)}\right)}\right| \\
\leq \sum_{\substack{p=0, p \neq q}}^{n-1}\left|\frac{t\left(\frac{\pi\left(\varphi_{p}-\varphi_{q}\right)}{2(n-1)}\right)^{2}(b-a+t)}{n t \sin ^{2}\left(\frac{\pi\left(\varphi_{p}-\varphi_{q}\right)}{2(n-1)}\right)}\right|+o(t),
\end{gathered}
$$

and, finally, we obtain

$$
\sum_{\substack{p=0, p \neq q}}^{n-1} \frac{1}{\sqrt{n}}\left|V\left(\varphi_{p}-\varphi_{q} ; a, b, t\right)\right| \leq \frac{d^{2} \gamma^{2}(b-a+t)}{n D^{2} \sin ^{2}(\gamma)}+o(t),
$$


where $\gamma=\frac{\pi D}{2(n-1)}$. This leads to the following estimates for the eigenvalues

$$
\begin{aligned}
& \lambda_{\max }(N) \leq \frac{n-1+t}{n}+\frac{d^{2} \gamma^{2}(n-1+t)}{n D^{2} \sin ^{2}(\gamma)}+o(t), \\
& \lambda_{\min }(N) \geq \frac{n-1-t}{n}-\frac{d^{2} \gamma^{2}(n-1-t)}{n D^{2} \sin ^{2}(\gamma)}-o(t) .
\end{aligned}
$$

Minimizing the first expression or maximizing the second with respect to $t$ we get

$$
\begin{aligned}
& \lambda_{\max }(N) \leq \frac{n-1}{n}+\frac{d^{2} \gamma^{2}(n-1)}{n D^{2} \sin ^{2}(\gamma)}, \\
& \lambda_{\min }(N) \geq \frac{n-1}{n}-\frac{d^{2} \gamma^{2}(n-1)}{n D^{2} \sin ^{2}(\gamma)} .
\end{aligned}
$$

Studying the asymptotic behaviour of the estimate for the smallest eigenvalue $\lambda_{\min }(N)$ we see that

$$
\lim _{n \rightarrow \infty}\left[\frac{n-1}{n}\left(1-\frac{d^{2} \gamma^{2}}{D^{2} \sin ^{2}(\gamma)}\right)\right]=\frac{D^{2}-d^{2}}{D^{2}}
$$

It follows that for the case of non-equidistant nodes, i.e. $D>d$, one has a more specific estimate for the smallest eigenvalue. In the case of the equidistant nodes, i.e. $D=d$, the estimate tells only that the smallest eigenvalue is greater or equal to zero, which is true by definition of a semi-positive definite matrix.

Coming back to the original matrix $F^{-1}$ we get the following estimate:

$$
\left\|F^{-1}\right\|_{2}=\sqrt{\lambda_{\min }(N)^{-1}} \leq \sqrt{\frac{n D^{2} \sin ^{2}(\gamma)}{(n-1)\left(D^{2} \sin ^{2}(\gamma)-d^{2} \gamma^{2}\right)}} .
$$

\subsubsection{Final Estimate for the Coupling Error}

Finally, estimate (3.4) has the form

$$
\begin{aligned}
& \left|f_{n}(\varphi)-\tilde{f}_{n}(\varphi)\right| \\
& \quad \leq \delta^{*} \sqrt{\frac{n D^{2} \sin ^{2}(\gamma)}{(n-1)\left(D^{2} \sin ^{2}(\gamma)-d^{2} \gamma^{2}\right)\left(\kappa^{2}-\kappa+\frac{5}{2}-\frac{1}{2} \sqrt{20 \kappa^{2}-20 \kappa+25}\right)}}\|\boldsymbol{\Phi}\|_{2} .
\end{aligned}
$$

It remains to estimate the norm of the vector of the basis functions $\Phi_{k}$. Taking into account that they are of the form

$$
\Phi_{k}(x)=e^{\frac{1}{2} i k x}
$$


we get

$$
\begin{aligned}
& \left|f_{n}(\varphi)-\tilde{f}_{n}(\varphi)\right| \\
& \quad \leq \sqrt{n} \delta^{*} \sqrt{\frac{n D^{2} \sin ^{2}(\gamma)}{(n-1)\left(D^{2} \sin ^{2}(\gamma)-d^{2} \gamma^{2}\right)\left(\kappa^{2}-\kappa+\frac{5}{2}-\frac{1}{2} \sqrt{20 \kappa^{2}-20 \kappa+25}\right)}} .
\end{aligned}
$$

To obtain the final estimate we need to notice that the error $\delta^{*}$ represents the $L_{2}$ error for displacements at the curved boundary of a coupling element $\mathbb{T}_{i}, i=1, \ldots, n-1$, which we denote by $\partial \mathbb{T}_{i}^{*}$, while the whole boundary of $\mathbb{T}_{i}$ is denoted in the standard way by $\partial \mathbb{T}_{i}$. According to the trace theorem for Lipschitz domains presented in [7] this error can be estimated as follows

$$
\|\mathbf{u}\|_{L_{2}\left(\partial \mathbb{T}_{i}^{*}\right)} \leq\|\mathbf{u}\|_{L_{2}\left(\partial \mathbb{T}_{i}\right)} \leq C\|\mathbf{u}\|_{W^{1,2}\left(\mathbb{T}_{i}\right)}
$$

where a constant $C$ depends only on $\mathbb{T}_{i}$. As it was shown in [12] over the coupling element $\mathbb{T}_{i}$ one can obtain the error estimate in $W^{1,2}\left(\mathbb{T}_{i}\right)$ by an extension of ideas from [5]. For the norm this error can be estimated as follows

$$
\left\|v-v_{h}\right\|_{1,2, \mathbb{T}_{i}} \leq C h_{\mathbb{T}}^{(i)}|v|_{2,2, \mathbb{T}_{i}}
$$

where $h_{\mathbb{T}}^{(i)}$ is a characteristic mesh constant of the specific coupling element define in (2.4) (see Fig. 4). Since we have made a rescaling of the interpolation nodes by $\frac{1}{\sqrt{n}}$ for the estimate of the spectral norm for the matrix $F^{-1}$, we need also to apply this rescaling to the characteristic mesh constant. Making the normalisation of the radius $r_{\mathrm{A}}$ and $l$ we obtain

$$
h_{\mathbb{T}}^{(i)}=\frac{1}{\sqrt{n}} \sqrt{\left(1+\frac{l}{n}\right)^{2} \tan ^{2}\left(\alpha_{i}\right)+\left(\frac{l}{n}\right)^{2}} .
$$

Thus we get the following estimate for $\delta^{*}$

$$
\delta^{*} \leq \sum_{i=1}^{n-1}\left[C_{i} \frac{1}{\sqrt{n}} \sqrt{\left(1+\frac{l}{n}\right)^{2} \tan ^{2}\left(\alpha_{i}\right)+\left(\frac{l}{n}\right)^{2}}|v|_{2,2, \mathbb{T}_{i}}\right],
$$

where constants $C_{i}$ depend only on $\mathbb{T}_{i}$.

Finally, the estimate for the coupling error is given in the following theorem:

Theorem 3.1 The coupling error at the interface $\Gamma_{\mathrm{AD}}$ can be estimated as follows

$$
\begin{aligned}
& \left|f_{n}(\varphi)-\tilde{f}_{n}(\varphi)\right| \\
& \leq \sum_{i=1}^{n-1} \sqrt{\frac{C_{i} n D^{2} \sin ^{2}(\gamma)\left[\left(1+\frac{l}{n}\right)^{2} \tan ^{2}\left(\alpha_{i}\right)+\left(\frac{l}{n}\right)^{2}\right]}{(n-1)\left(D^{2} \sin ^{2}(\gamma)-d^{2} \gamma^{2}\right)\left(\kappa^{2}-\kappa+\frac{5}{2}-\frac{1}{2} \sqrt{20 \kappa^{2}-20 \kappa+25}\right)}}|v|_{2,2, \mathbb{T}_{i}},
\end{aligned}
$$


where $n$ is a number of interpolation nodes, $D$ is the maximum distance between two nodes, $d$ is the minimal distance between two nodes, $\alpha_{i}, i=1, \ldots, n-1$ are angles defined by geometry of each coupling element, and $\gamma=\frac{\pi D}{2(n-1)}$.

The estimate (3.11) covers the general case of nodes distribution. In practice it means, that for a given triangulation $\mathcal{F}_{h}$ we obtain a specific estimate from this general case, i.e. for a fixed set of angles $\alpha_{1}, \alpha_{2}, \ldots, \alpha_{n-1}$.

Studying the asymptotic behaviour of the estimate (3.11) for a general $\alpha$ and taking into account that with an increasing number $n$ this angle decreases proportionally due to construction, as well as a size of $\mathbb{T}$ decreases, i.e. the semi-norm vanishes, we see that

$$
\lim _{n \rightarrow \infty} \sqrt{\frac{n D^{2} \sin ^{2}(\gamma)\left[\left(1+\frac{l}{n}\right)^{2} \tan ^{2}(\alpha)+\left(\frac{l}{n}\right)^{2}\right]}{(n-1)\left(D^{2} \sin ^{2}(\gamma)-d^{2} \gamma^{2}\right)\left(\kappa^{2}-\kappa+\frac{5}{2}-\frac{1}{2} \sqrt{20 \kappa^{2}-20 \kappa+25}\right)}}=0 .
$$

The constructed estimate for the coupling error converges to zero approximately as $O\left(\frac{1}{n}\right)$ for all possible values of the material parameter $\kappa \in(1,3)$.

\section{Conclusions}

In this paper we have presented error estimates for a continuous coupling of an analytical and a finite element solution. The question of quality of the coupling remained open from previous research on such types of coupling. The coupling error plays an important role in a numerical realisation of the method, especially if an analytical element $\mathbb{A}$ of a fixed size is considered. In this case a refinement with a global scaling factor is not possible, and due to a fixed size of the analytical element the classical theory of the finite element method cannot be used. To obtain the error estimate in $\mathbb{A}$ we have considered the question of approximation by interpolation, which leads to the estimate in two parts: the interpolation error and the coupling error. Such an approach allows to underline clearly the effect of the coupling error. The estimation of this coupling error is based on the estimation of the spectral norm of the corresponding interpolation matrix. The constructed estimate converges to zero with an increasing number of the interpolation nodes at the coupling interface $\Gamma_{\mathrm{AD}}$, i.e. with an increasing number of coupling elements.

Finally we would like to mention the following important questions for future research:

(i) Finding the optimal position of interpolation nodes at the coupling interface $\Gamma_{\mathrm{AD}}$ in order to get a sharper estimate for the coupling error $\left|f_{n}(\varphi)-\tilde{f}_{n}(\varphi)\right|$. This question leads us directly to a relation between local and global error estimates, because by using a non-equidistant node distribution on one hand we improve the quality of the coupling error (local estimate), but on another hand we disturb the symmetry of the finite element mesh, which in general leads to higher error in the finite element approximation (global estimate). 
(ii) Find the optimum ratio between a number of the interpolation nodes and a size of the radius of the analytical element, this ratio is expected to be a domain dependent.

Acknowledgements The research of U. Kähler is supported by Portuguese funds through the CIDMA, Center for Research and Development in Mathematics and Applications, and the Portuguese Foundation for Science and Technology ("FCT-Fundação para a Ciência e a Tecnologia"), within project UID/MAT/04106/2013. The research of D. Legatiuk is supported by the German Research Foundation (DFG) via Research Training Group "Evaluation of Coupled Numerical Partial Models in Structural Engineering (GRK 1462)", which is gratefully acknowledged.

\section{References}

1. Bock, S., Gürlebeck, K., Legatiuk, D.: On a special finite element based on holomorphic functions. AIP Conf. Proc. 1479, 308 (2012)

2. Bock, S., Güerlebeck, K.: On a spatial generalization of the Kolosov-Muskhelishvili formulae. Math. Methods Appl. Sci. 32, 223-240 (2009)

3. Bock, S.: On monogenic series expansions with applications to linear elasticity. Adv. Appl. Clifford Algebras 24, 931-943 (2014)

4. Bock, S., Gürlebeck, K., Legatiuk, D., Nguyen, H.M.: $\psi$-Hyperholomorphic functions and a KolosovMuskhelishvili formula. Math. Methods Appl. Sci. 38, 5114-5123 (2015)

5. Ciarlet, P.G.: The Finite Element Method for Elliptic Problems. North-Holland, Amsterdam (1978)

6. Davis, P.J.: Interpolation and Approximation. Dover, New York (1975)

7. Ding, Z.: A proof of the trace theorem of Sobolev spaces on Lipschitz domains. Proc. Am. Math. Soc. 123(2), 591-600 (1996)

8. Ferreira, P.J.S.G.: Superresolution, the recovery of missing samples and Vandermonde matrices on the unit circle. In: Proceedings of the 1999 Workshop on Sampling Theory and Applications, Loen (1999)

9. Gaier, D.: Lecture on Complex Approximation. Birkhäuser, Boston (1987)

10. Gürlebeck, K., Legatiuk, D.: On the continuous coupling of finite elements with holomorphic basis functions. Hypercomplex Analysis: New Perspectives and Applications. Birkhäuser, Basel. ISBN 9783-319-08770-2

11. Gürlebeck, K., Kähler, U., Legatiuk, D.: Interpolation problem arising in a coupling of finite element method with holomorphic basis functions. AIP Conf. Proc. 1648 (2015). doi:10.1063/1.4912655

12. Legatiuk, D., Nguyen, H.M.: Improved convergence results for the finite element method with holomorphic functions. Adv. Appl. Clifford Algebra (2014). doi:10.1007/s00006-014-0501-1

13. Legatiuk, D.: Evaluation of the coupling between an analytical and a numerical solution for boundary value problems with singularities. Ph.D. thesis (2015). ISBN: 978-3-95773-193-7

14. Liebowitz, H.: Fracture, an advanced treatise. Mathematical Fundamentals, vol. II. Academic Press, London (1968)

15. Lurie, A.I.: Theory of Elasticity. Foundations of Engineering Mechanics. Springer, Berlin (2005) (translated from Russian)

16. Mußchelischwili, N.I.: Einige Grundaufgaben der mathematischen Elastizitätstheorie. VEB Fachbuchverlag Leipzig (1971)

17. Piltner, R.: Special finite elements with holes and internal cracks. Int. J. Numer. Methods Eng. 21, 509-528 (1985)

18. Piltner, R.: The derivation of special purpose element functions using complex solution representation. Comput. Assist. Mech. Eng. Sci. 10(4), 597-607 (2003)

19. Piltner, R.: Some remarks on finite elements with an elliptic hole. Finite Elements Anal. Design 44(1213), 767-772 (2008)

20. Schumaker, L.: Spline Functions on Triangulation. Cambridge University Press, Cambridge (2007) 